\title{
Indicadores da Qualidade dos Registros e da Assistência Ambulatorial em Maringá, (Estado do Paraná, Brasil), 1991: Um Exercício de Avaliação
}

\author{
Indicators of Quality for Medical Records and Ambulatory Care in Maringá (State of \\ Paraná, Brazil), 1991: An Exercise in Evaluation
}

Maria José Scochi ${ }^{1}$

SCOCHI, M. F. Indicators of Quality for Medical Records and Ambulatory Care in Maringá (State of Paraná, Brazil), 1991: An Exercise in Evaluation. Cad. Saúde Públ., Rio de Faneiro, 10 (3): 356-367, ful/Sep, 1994.

This study sought to establish health-care quality indicators based on data from routine clinical records produced by the Municipal Health Department in Maringá, Paraná State. Records for 7813 patients seen in the month of October 1991 at three Integrated Health Centers (IHC) were assessed to determine whether the recorded information allowed for the formulation of quality indicators. Indicators of data quality, demand, coverage, resolubility, and geographic distribution of the clientele by IHC were formulated. Some $49.7 \%$ of records had no diagnostic hypothesis, and $14.8 \%$ of recorded diagnoses were illegible, $78 \%$ of visits were by residents of the IHCs' cachement areas; there was a low estimated coverage of pre-natal care of $37 \%$; an estimated $70 \%$ of children under one year of age residing in the area were seen during the month; patients seen in October 1991 had a mean of 3 physician visits per person during the preceding year and $4.3 \%$ of records specified a referral to another service. Patient records in the centers studied are precarious as information services for either routine internal use or external evaluation. In spite of these deficiencies, it was possible to extract some indicators of quality; however, significant improvements in recording are needed to support more in-depth evaluation.

Key words: Health Planning; Evaluation of Health Services; Quality; Health Information; Ambulatory Care

\section{INTRODUÇÃO}

A municipalização está trazendo inovações no campo da organização dos serviços de saúde para alguns municípios e, com ela, a possibilidade da criação de formas diferenciadas de intervenção. A Constituição de 1988 e sua subseqüente regulamentação que contempla, entre outros requisitos, a obrigatoriedade de elaboração de planos municipais de assistência à saúde, favorecem os processos de inovação a nível local.

\footnotetext{
${ }^{1}$ Departamento de Enfermagem e Obstetrícia da Universidade Estadual de Campinas. Avenida Colombo, 3690, Maringá, PR, 87020-250, Brasil.
}

O cumprimento do preceito legal requer dos departamentos ou secretarias municipais de saúde a utilização do planejamento, ciência esta que depende da avaliação do que é realizado. A avaliação, antes na prática quase inexistente, torna-se hoje preocupação das equipes responsáveis pela programação dos serviços, possibilitando o desenvolvimento de análises críticas do que e como são ofertados os serviços de saúde à população.

Entendendo a avaliação como um dos componentes da garantia da qualidade dos serviços, junto à equipe de programação e avaliação dos serviços de saúde da Secretaria Municipal de Saúde de Maringá (SMSM), resolveu-se enveredar por esse campo de investigação. 


\section{UMA PRIMEIRA APROXIMAÇÃO CONCEITUAL}

De acordo com Vuori (1982: 19), garantia de qualidade pode ser definida como " $a$ medida do nível atual da qualidade dos serviços prestados mais os esforços para modificar, quando necessário, a provisão desses serviços à luz dos resultados $d a$ medida"; donde pode-se depreender que a garantia da qualidade consiste de dois componentes: um analítico - medida ou avaliação da qualidade - e um corretivo.

O primeiro dos componentes nos remete para uma das primeiras dificuldades a ser enfrentada na investigação - a definição conceitual. Pois, ao se propor tratar de investigar avaliação de qualidade, o que se entende por qualidade? Como e para que esta deve ser definida e medida?

Concorda-se com alguns autores (Donabedian, 1984; Silver, 1992a; Vuori, 1982) que o conceito de qualidade é largamente utilizado e é uma busca constante dos indivíduos. No campo da atenção à saúde existem diversas ópticas para sua apreciação, a maioria sendo pouco operacionalizada, o que leva a existência de definições múltiplas e conflitivas.

Para Donabedian (1984), por exemplo, a busca da definição de qualidade, ainda que se inicie em seu módulo mais simples da atenção, isto é, o tratamento que proporciona um médico ou outro profissional a um episódio de enfermidade claramente definido em um paciente dado, é possível dividir este tratamento em dois aspectos, o técnico e o interpessoal, cada um sugerindo existência de diferentes conceitos de qualidade.

Em relação ao segundo componente de garantia da qualidade - o corretivo - pode-se dizer que este, talvez, seja o objetivo primeiro que deve preceder qualquer propósito de avaliação, isto é, apontar as possibilidades de intervenção na realidade com a finalidade de promover mudanças.

No contexto da Reforma Sanitária Brasileira a avaliação do serviço também poderá responder a indagação de qual modelo assistencial a municipalização estará ajudando a implementar: é um modelo assistencial hegemônico, privativista, hospitalizante, que privilegia as ações médicas individuais, reproduzindo as tendências anteriores do sistema de saúde? Ou constitui-se de fato em um modelo alternativo que operacionalize as diretrizes de regionalização, hierarquização, universalização, e a ênfase na prevenção, definidas na VIII Conferência Nacional de Saúde (CNS) (Cordoni Jr. \& Bertone, 1992)?

Para esta análise é indispensável desenvolver processos avaliativos que contenham diversas fontes de informação sobre o sistema de saúde. Segundo Rocha et al. (1991: 186) “a viabilização de um sistema público de saúde requer amplos estudos epidemiológicos e de serviços de saúde os quais deverão ter base em eficientes sistemas de informação".

Fala-se, por exemplo, que existe dificuldade em saber da demanda populacional às unidades e do conteúdo assistencial, uma vez que informações relativas as mesmas geralmente são elaboradas, a partir de observações empíricas da equipe. O prontuário médico, universalmente identificado como uma das fontes mais importantes, é pouco utilizado. A justificativa é que para verificar as características observadas seria necessário realizar pesquisas especiais, pois desconfia-se da qualidade com que os dados são registrados rotineiramente. Apesar disto, os dados continuam a ser registrados da mesma forma. Entende-se que esse tipo de conduta é obstáculo ao processo de implementação de garantia da qualidade, pois não confiando-se nos dados registrados por que continua-se a registrá-los? Por que, ainda, continua-se reformulando os prontuários sem uma avaliação efetiva dos mesmos e continua-se exigindo dos trabalhadores que os utilizem?

Às vezes a qualidade da informação é proporcional à qualidade da assistência, mas na ausência do registro da informação é difícil fazer inferências sobre a qualidade. Não se pretende fazer apologia da informação como solução dos problemas vividos pela rede de assistência e, tampouco, afirmar que sem informação não se pode modificar o que aí está posto. Mas ressaltar que as informações existentes precisam ser conhecidas e analisadas, para aprimorar a qualidade da assistência.

A gerência subsidiada na informação é mais que uma necessidade, uma nova filosofia mundial de trabalho. Os avanços na organização da 
assistência serão baseados, cada vez mais, em uma informação descentralizada e disponível, propiciando aos trabalhadores a melhoria de seu processo de trabalho e à sociedade a possibilidade de entendimento e controle através de suas organizações (Médici, 1991).

No levantamento bibliográfico realizado observou-se que nos anos mais recentes, apesar da importância das fontes de informação para a gerência dos serviços ambulatoriais, são poucos os artigos dedicados à avaliação da qualidade dos registros em prontuários, entre estes destacam-se os trabalhos de Lotufo \& Duarte (1987) e Modesto et al. (1992).

O primeiro analisa informações sobre a oferta dos serviços de saúde no município de Cáceres, Mato Grosso, no período de 1981 a 1984. Os resultados revelaram que apesar da expansão da rede básica dos serviços de saúde, em função da implantação do Programa Rural Integrado (PDRI), não se observou alterações significativas na qualidade da assistência à saúde da população. Em relação à qualidade dos registros constatou uma elevada freqüência de diagnósticos ilegíveis, incoerências de registros e ausência de diagnóstico.

Modesto et al. (1992) realiza um levantamento dos arquivos médicos no Distrito Sanitário de Itapagipe, Salvador (BA), nos anos de 1986 e 1989, objetivando avaliar a qualidade dos registros antes e depois da Reforma Sanitária do Sistema Unificado Descentralizado de Saúde (SUDS), iniciado em 1987. Observa que após a implantação do SUDS houve uma melhora na padronização dos registros, no entanto, essa não foi acompanhada por uma melhoria na qualidade do preenchimento. Afirma que "mesmo após a implantação do SUDS os médicos envolvidos na atenção primária à saúde continuam preenchendo insatisfatoriamente os prontuários, apesar de se registrar maior volume de diagnósticos e menor proporção de prescrição de medicamentos" (Modesto et al., 1992: 67).

O objetivo do presente estudo foi conhecer o tipo de registro realizado em prontuários em Núcleos Integrados de Saúde (NIS) no município de Maringá (PR) e se o que é registrado permite uma primeira aproximação da avaliação de qualidade, que possam contribuir para as atividades de garantia da qualidade a serem desenvolvidas.

\section{MATERIAL E MÉTODOS}

Maringá, município com 240 mil habitantes (IBGE, 1991) sofreu uma expansão da oferta de serviços básicos de saúde, passando de quatro unidades sanitárias, em 1982, a vinte e quatro Núcleos Integrados de Saúde (NIS), em 1992. Essas unidades, funcionando doze horas diárias, ofertam serviços básicos de saúde, incluindo atenção médica em cada especialidade básica, atendimentos de enfermagem, odontologia e psicologia.

A investigação foi realizada em três NIS. O NIS Alvorada I, localizado num bairro de maior densidade populacional, estimada em $23 \mathrm{mil}$ habitantes, apresentava um grande volume de atendimento e possuía uma equipe profissional de nove médicos, uma enfermeira, oito auxiliares de enfermagem, três dentistas, um psicólogo, um auxiliar administrativo e duas zeladoras. O NIS Cidade Alta atende uma população, adscrita a um conjunto habitacional, com 5.300 habitantes e contava com equipe profissional mínima, composta de três médicos, uma enfermeira, três auxiliares de enfermagem, dois dentistas, um auxiliar administrativo e uma zeladora. O NIS Vila Operária situa-se, comparativamente, em situação intermediária entre os primeiros, isto é, contava com uma equipe profissional de cinco médicos, uma enfermeira, quatro auxiliares de enfermagem, três dentistas, um psicólogo que atua, também, em outra unidade, um auxiliar administrativo e uma zeladora, atendendo uma população aproximada de 10.930 habitantes. A seleção procurou retratar unidades distintas do ponto de vista de volume de atendimento e capacidade física instalada, sem ser representativa da rede como um todo.

Após um levantamento preliminar do registro em 65 prontuários de um dos Núcleos, observou-se a ausência de informações elementares e legíveis sobre o cliente atendido, tais como, data de nascimento, endereço etc. Além disso, uma alta freqüência de anotações ilegíveis onde não se conseguia identificar o motivo de procura pelo atendimento, as principais hipóteses diagnósticas (HD) ou procedimentos realizados.

Estes achados preliminares levaram a uma primeira intervenção, que constituiu-se de 
reuniões com as três equipes profissionais, onde foram discutidos os objetivos do projeto e era solicitada a colaboração no registro adequado de informações, pelo menos durante o período em que os dados seriam coletados. Tentou-se vencer a resistência de alguns, estimulando-os a ver o quão inócuo e alienante é o trabalho realizado e que, ao final da jornada diária, as informações registradas não permitem verificar o que acontece na unidade, quais patologias são mais freqüentes e se têm ou não resolutividade as ações ali realizadas. Além disso, discutiu-se que o registro da informação de forma legível é parte e dever do trabalho nas unidades onde as atividades devem, em tese, ser desenvolvidas por equipes multiprofissionais.

Realizaram-se, também, reuniões com as equipes médicas, para solicitar o registro numérico de até três hipóteses diagnósticas de acordo com a Classificação Internacional de Doenças (CID), $9^{\mathrm{a}}$ revisão. Apesar de fornecer a cada profissional um exemplar da CID, alguns alegaram falta de tempo para procurar o número relativo a $\mathrm{HD}$, a estes solicitou-se, então, o registro nominal da HD de forma legível para que as estagiárias do curso de Enfermagem procedessem a codificação. A codificação prevista pela equipe não aconteceu, já que as HD registradas nominalmente estavam ilegíveis.

Após esta intervenção foi realizado um levantamento de todos os prontuários de usuários atendidos nas três unidades durante o mês de outubro de 1991. A unidade de análise foi o atendimendo realizado, já que se pretendia retratar a demanda à unidade e não as características dos indivíduos. Isto é, nos casos em que um indivíduo compareceu a unidade mais de uma vez no mês, os dados referentes a cada atendimendo foram contabilizados, salvo as tabelas relativas à cobertura ambulatorial.

A coleta das informações visou obter dados relativos ao mínimo de informações esperadas para cada cliente, ou seja, sexo, idade, procedência, número total de consultas médicas, de procedimentos médicos e de enfermagem, consultas de enfermagem, consultas de psicologia e de odontologia, entre 01 de outubro de 1990 e 30 de outubro de 1991; principais hipóteses diagnósticas, encaminhamentos internos ou externos realizados registrados na última consulta.

As informações foram coletadas por oito estagiárias do curso de Enfermagem. Pesquisou-se 7.813 prontuários correspondendente $\mathrm{a}$, aproximadamente, $10 \%$ do volume de atendimentos/ mês realizados na rede municipal de serviços de saúde. No NIS Alvorada I foram obtidas informações de 4.567 (58,47\%) prontuários, no NIS Vila Operária, 1.961 (25,09\%) e no da Cidade Alta, 1.285 (16,44\%).

\section{RESULTADOS}

\section{Qualidade do Registro}

Como pode ser observado na Tabela 1, após a intervenção, informações relativas ao cliente atendido, tais como: data de nascimento, número de prontuário, sexo e endereço estiveram presentes na quase totalidade dos prontuários das três unidades, sendo que a unidade Alvorada I apresenta uma freqüência menor de registro de informações básicas sobre o usuário, quando comparada com as outras duas unidades.

Por se tratar de unidade ambulatorial onde existe a possibilidade de mais de uma hipótese diagnóstica, buscou-se contabilizar no mínimo três. Observou-se, no entanto, que os prontuários apresentam, em sua maioria, uma única hipótese diagnóstica em cada consulta analisada. E essas foram registradas, na última consulta médica em $49,13 \%$ dos atendimentos, sendo que dessas, $34,33 \%$ foram codificadas pelo médico e $14,80 \%$ estavam ilegíveis. Duas hipóteses diagnósticas foram registradas em apenas $2,65 \%$ e $0,20 \%$ dos atendimentos apresentavam três. A unidade Alvorada I apresentou menor percentual de registro de HD e de legibilidade (Tabela 2).

\section{Procedência da Clientela}

Apesar de não estar claro para os profissionais e usuários qual é de fato a área de abrangência populacional adscrita às unidades sanitárias, alguns profissionais afirmam, freqüentemente, que muitos usuários não residem na área de abrangência das mesmas, o que é contraposto pelo levantamento realizado. Pode-se obser- 
var que $78,02 \%$ dos atendimentos prestados foram destinados a moradores que residem na área de abrangência, 20,28\% de locais próximos a outros núcleos integrados e $1,70 \%$ a moradores de municípios vizinhos de Maringá. Ocorre pouca variação nas freqüências quando se analisa a procedência do cliente por unidade pesquisada (Tabela 3).

TABELA 1. Freqüência (\%) de Registro de Data de Nascimento, Número de Prontuário, Sexo e Endereço em Prontuários nos Atendimentos Realizados pelos Núcleos Integrados de Saúde, Maringá, em Outubro 1991

\begin{tabular}{lcccc}
\hline \hline & $\begin{array}{c}\text { Data de } \\
\text { Nascimento }\end{array}$ & $\begin{array}{c}\mathrm{N}^{\mathrm{o}} \\
\text { de Prontuário }\end{array}$ & Sexo & Endereço \\
\cline { 2 - 5 } NIS & $\begin{array}{c}\text { Sem Registro } \\
(\%)\end{array}$ & $\begin{array}{c}\text { Sem Registro } \\
(\%)\end{array}$ & $\begin{array}{c}\text { Sem Registro } \\
(\%)\end{array}$ & $\begin{array}{c}\text { Sem Registro } \\
(\%)\end{array}$ \\
\hline $\begin{array}{l}\text { Alvorada I } \\
(\mathbf{n}=4.567)\end{array}$ & 3,24 & 1,07 & 0,52 & 0,76 \\
$\begin{array}{l}\text { Vila Operária } \\
(\mathbf{n}=1.961)\end{array}$ & 1,20 & 0,10 & 0,15 & 0,76 \\
$\begin{array}{l}\text { Cidade Alta } \\
(\mathbf{n}=1.285)\end{array}$ & 1,32 & 0,00 & 0,15 & 0,85 \\
\hline $\begin{array}{l}\text { Total } \\
(\mathrm{N}=7.813)\end{array}$ & 2,40 & 0,65 & 0,37 & 0,78 \\
\hline \hline
\end{tabular}

TABELA 2. Freqüência (\%) de Registro de Hipótese Diagnóstica na Última Consulta Médica dentre os Atendimentos Realizados pelos Núcleos Integrados de Saúde, Maringá, em Outubro 1991

\begin{tabular}{lccc}
\hline \hline & \multicolumn{2}{c}{ Freqüência do Registro de Hipótese Diagnóstica } \\
\cline { 2 - 4 } & HD & \multicolumn{2}{c}{ HD Registradas } \\
\cline { 2 - 4 } NIS & $\begin{array}{c}\text { Sem Registro } \\
(\%)\end{array}$ & $\begin{array}{c}\text { Legível } \\
(\%)\end{array}$ & $\begin{array}{c}\text { Ilegível } \\
(\%)\end{array}$ \\
\hline $\begin{array}{l}\text { Alvorada I } \\
(\mathbf{n}=3.738)\end{array}$ & 47,22 & 34,62 & 18,16 \\
$\begin{array}{l}\text { Vila Operária } \\
(\mathbf{n}=1.705)\end{array}$ & 34,13 & 45,40 & 20,47 \\
$\begin{array}{l}\text { Cidade Alta } \\
(\mathbf{n}=1.045)\end{array}$ & 31,77 & 57,42 & 10,81 \\
\hline $\begin{array}{l}\text { Total } \\
(\mathrm{N}=6.488)\end{array}$ & 41,29 & 41,12 & 17,59 \\
\hline \hline
\end{tabular}


TABELA 3. Procedência dos Usuários por Atendimentos Realizados pelos Núcleos Integrados de Saúde, Maringá, em Outubro 1991

\begin{tabular}{lccc}
\hline \hline & \multicolumn{3}{c}{ Procedência do Usuário } \\
\cline { 2 - 4 } & $\begin{array}{c}\text { Área Adscrita } \\
\text { a Unidade } \\
\text { NIS }\end{array}$ & $\begin{array}{c}\text { Área Adscrita } \\
\text { a Outra Unidade } \\
(\%)\end{array}$ & $\begin{array}{c}\text { Pertencente a } \\
\text { Municípios Vizinhos } \\
(\%)\end{array}$ \\
\hline $\begin{array}{l}\text { Alvorada I } \\
(\mathbf{n}=4.567)\end{array}$ & 79,54 & 19,19 & 1,37 \\
$\begin{array}{l}\text { Vila Operária } \\
(\mathbf{n}=1.961)\end{array}$ & 75,67 & 22,28 & 2,05 \\
$\begin{array}{l}\text { Cidade Alta } \\
(\mathbf{n}=1.285)\end{array}$ & 76,18 & 21,08 & 2,74 \\
\hline $\begin{array}{l}\text { Total } \\
(\mathrm{N}=7.813)\end{array}$ & 78,02 & 20,28 & 1,70 \\
\hline \hline
\end{tabular}

\section{Características dos Usuários}

$\mathrm{Na}$ análise das hipóteses diagnósticas que estavam legíveis em $34,33 \%$ dos prontuários, pode-se observar que as cinco mais freqüentes são relativas às infecções de vias aéreas superiores, $15,09 \%$; às afecções intestinais, $4,11 \%$; à hipertensão arterial, 4,02\%; às afecções genito-urinárias, 3,98\%; aos distúrbios gástricos, $3,66 \%$. Esses percentuais não variaram quando se analisou as unidades isoladamente.

Em relação ao sexo e idade dos usuários constatou-se a predominância de mulheres, $60,25 \%$ contra $39,75 \%$ de homens. A demanda se divide entre adultos de 15 - 45 anos, beneficiários de um terço dos atendimentos, crianças menores de 15 anos constituíram 44,87\%, 12,91\% dos usuários atendidos tinham de 45 a 65 anos e apenas 5,36\% dos atendimentos foram destinados a usuários com mais de 65 anos (Tabela 4).

\section{Cobertura nas Unidades}

A população de menores de um ano em 1990, de acordo com o Instituto Paranaense de Desenvolvimento (Ipardes, 1990), foi 1,8\% da população total. Com base neste dado, estima-se que o número de gestantes esperadas no ano de 1991, seja algo em torno de 2,5\% da população. Aplicando este percentual à população de cada unidade espera-se entre a população de abrangência do NIS Alvorada I, 575 gestantes, para o de Vila Operária, 270 e Cidade Alta, 132 por ano. Considerando o período gestacional, esperava-se que a cada mês as unidades idealmente atendessem $2 / 3$ do número de gestantes esperadas por ano, ou seja, cada uma deveria passar, após o segundo mês de gestação, oito vezes ao ano pela consulta médica. O levantamento sobre o número de gestantes atendidas revelou uma cobertura estimada baixa e diferenciada para as três unidades (Tabela 5).

Aplicando o percentual de 1,8 \% à população total de cada unidade obteve-se o número estimado de crianças esperadas (Tabela 6). Considerando que o Programa de Assistência Integral à Saúde da Criança do Ministério da Saúde (1984) recomenda seis consultas no primeiro ano de vida, estimou-se que metade destas crianças deveriam ser consultadas no mês, ou seja a criança receberia pelo menos uma consulta a cada dois meses, com esse cálculo o Alvorada I deveria atender 207 crianças; o Vila Operária, 99 e o de Cidade Alta, 48 . O levantamento do número de crianças menores de um ano, atendidas nas três unidades revelou uma freqüência de atendimendo acima destes valores no mês nas unidades de Vila Operária e Cidade Alta, no Alvorada I encontrou-se valores próximo às normas. 
TABELA 4. Faixa Etária e Sexo de Usuários por Atendimento Realizado pelos Núcleos Integrados de Saúde, Maringá, em Outubro 1991

\begin{tabular}{|c|c|c|c|c|c|c|c|c|}
\hline \multirow[b]{2}{*}{$\begin{array}{l}\text { Faixa } \\
\text { Etária }\end{array}$} & \multicolumn{2}{|c|}{ Alvorada I } & \multicolumn{2}{|c|}{ Vila Operária } & \multicolumn{2}{|c|}{ Cidade Alta } & \multicolumn{2}{|c|}{ Total } \\
\hline & $\begin{array}{c}\text { Fem. } \\
(\%)\end{array}$ & $\begin{array}{c}\text { Masc. } \\
(\%)\end{array}$ & $\begin{array}{c}\text { Fem. } \\
(\%)\end{array}$ & $\begin{array}{c}\text { Masc. } \\
(\%)\end{array}$ & $\begin{array}{c}\text { Fem. } \\
(\%)\end{array}$ & $\begin{array}{c}\text { Masc. } \\
(\%)\end{array}$ & $\begin{array}{c}\text { Fem. } \\
(\%)\end{array}$ & $\begin{array}{c}\text { Masc. } \\
(\%)\end{array}$ \\
\hline$<1$ Ano & 10,06 & 6,08 & 9,53 & 8,16 & 8,48 & 9,03 & 11,24 & 11,05 \\
\hline $01-05$ & 4,69 & 5,80 & 6,48 & 6,48 & 5,05 & 6,23 & 4,63 & 5,30 \\
\hline $05-15$ & 6,43 & 6,43 & 6,63 & 6,83 & 7,24 & 7,94 & 6,39 & 6,26 \\
\hline $15-45$ & 26,06 & 10,63 & 26,77 & 8,82 & 29,96 & 9,34 & 26,75 & 9,85 \\
\hline $45-65$ & 9,15 & 4,60 & 9,38 & 4,38 & 6,77 & 4,20 & 8,40 & 4,50 \\
\hline $65 \mathrm{e}+$ & 3,50 & 2,82 & 2,86 & 2,45 & 1,71 & 2,72 & 2,83 & 2,38 \\
\hline Total & 58,89 & 36,38 & 61,65 & 37,12 & 59,21 & 39,46 & 60,25 & 39,75 \\
\hline
\end{tabular}

TABELA 5. Número de Gestantes Esperadas por Mês, Atendidas na Área de Abrangência e Cobertura Estimada por Núcleo Integrado de Saúde, Maringá, em Outubro 1991

\begin{tabular}{|c|c|c|c|}
\hline \multirow[b]{2}{*}{ NIS } & \multicolumn{3}{|c|}{ Gestantes Esperadas, Atendidas e Cobertura } \\
\hline & $\begin{array}{c}\text { Gestantes } \\
\text { Esperadas por Mês } \\
\mathrm{N}^{\mathrm{o}}\end{array}$ & $\begin{array}{c}\text { Gestantes } \\
\text { Atendidas } \\
\mathrm{N}^{\mathrm{o}}\end{array}$ & $\begin{array}{c}\text { Cobertura } \\
\text { Estimada por Mês } \\
\mathrm{N}^{\mathrm{o}}\end{array}$ \\
\hline Alvorada I & 431 & 58 & 13,46 \\
\hline Vila Operária & 205 & 51 & 24,88 \\
\hline Cidade Alta & 99 & 39 & 39,39 \\
\hline Total & 735 & 241 & 32,79 \\
\hline
\end{tabular}

TABELA6. Número Estimado de Crianças Menores de um Ano, Atendidas no Mês na Área de Abrangência eCobertura Estimada por Núcleo Integrado de Saúde, Maringá, em Outubro 1991

\begin{tabular}{lccc}
\hline \hline & \multicolumn{3}{c}{ Gestantes Menores de um Ano Esperadas, Atendidas e Cobertura } \\
\cline { 2 - 4 } & $\begin{array}{c}\text { Estimativa de } \\
\text { Crianças < de 1 Ano }\end{array}$ & $\begin{array}{c}\text { Crianças < de 1 Ano } \\
\text { Atendidas no Mês }\end{array}$ & $\begin{array}{c}\text { Cobertura } \\
\text { Estimada no Mês } \\
\text { NIS }\end{array}$ \\
$\mathrm{N}^{\text {* }}$ & $\mathrm{N}^{\mathbf{0}}$ & $(\%)$ \\
\hline Alvorada I & 414 & 177 & 42,75 \\
Vila Operária & 198 & 127 & 64,14 \\
Cidade Alta & 96 & 65 & 67,71 \\
\hline Total & 708 & 366 & 51,17 \\
\hline \hline
\end{tabular}

* Apenas aquelas moradoras na área de abrangência nascidas a partir de 15/10/90.

${ }^{\star \star}$ Cobertura calculada como

$N^{o}$ de crianças < de 1 ano atendidas no mês

$N^{o}$ de crianças < de 1 ano estimadas na área de abrangência 
As pessoas atendidas em outubro tiveram, em média, três consultas médicas no ano nas três unidades, esta concentração repete-se para cada uma delas em separado. Na Tabela 7, observa-se que as unidades apresentam percentuais semelhantes na concentração de consultas médicas.

Verificou-se também a concentração durante o mês em que se realizou a coleta dos dados; para os três núcleos foi observado que cerca de $25 \%$ dos clientes receberam mais de uma consulta naquele mês, $8,32 \%$ receberam mais de três. No NIS Alvorada I, por exemplo, uma usuária de 33 anos recebeu dezenove consultas médicas durante o mês e em nenhuma delas foi registrada qualquer hipótese diagnóstica.

Assim como se verificou a concentração de consultas médicas, verificou-se também a freqüência com que aparece o registro no prontuário do número de atendimentos reali- zados exclusivamente pelo enfermeiro, ou seja, consulta de enfermagem (CE); estas aparecem em apenas $10,02 \%$ dos mesmos no total e não apresenta diferenças quando se analisa o dado para as unidades em separado.

Dos registros levantados sobre atendimentos de enfermagem (por exemplo, pré e pós consultas, curativos, aplicação de medicamentos, entre outros) que deveriam fazer parte de qualquer atendimento, verificou-se que estes aparecem em apenas $74,84 \%$ dos prontuários e que $20,98 \%$ dos usuários receberam mais de um atendimento de enfermagem. Esperava-se dos procedimentos registrados, no mínimo, o dobro das consultas, pois para cada consulta, estão previstos na programação um atendimento de pré e pós consulta. As pessoas atendidas em outubro de 1991 tiveram 6.488 consultas médicas, 1.083 consultas de enfermagem e 6.006 procedimentos de enfermagem.

TABELA 7. Freqüência(\%) de Consultas Médicas no Último Ano das Pessoas Atendidas pelos Núcleos

\begin{tabular}{lccccc}
\hline \hline & \multicolumn{5}{c}{ Concentração de Consultas Médicas } \\
\hline NIS & $\begin{array}{c}\text { Sem Consulta } \\
(\%)\end{array}$ & $\begin{array}{c}1 \text { Consulta } \\
(\%)\end{array}$ & $\begin{array}{c}2 \text { Consultas } \\
(\%)\end{array}$ & $\begin{array}{c}3 \text { Consultas } \\
(\%)\end{array}$ & $\begin{array}{c}+ \text { de } 4 \\
(\%)\end{array}$ \\
\hline Alvorada I & 18,14 & 30,98 & 17,07 & 10,32 & 23,41 \\
Vila Operária & 13,05 & 25,70 & 15,19 & 12,08 & 33,96 \\
Cidade Alta & 18,75 & 21,63 & 16,57 & 11,83 & 31,31 \\
\hline Total & 16,62 & 27,86 & 16,52 & 11,01 & 27,99 \\
\hline \hline
\end{tabular}

\section{"Resolutividade" do Primeiro Nível da Atenção}

Em relação ao número de encaminhamentos, observou-se que, no total, $4,31 \%$ dos clientes atendidos tiveram um encaminhamento formal para outros serviços registrados no prontuário. No Alvorada I foi de 4,09; Vila Operária de 3,77 e no da Cidade Alta de 5,76\%.

\section{DISCUSSÃO}

A análise dos dados que foi realizada no conjunto das três unidades e para cada uma isoladamente, revelou que as formas utilizadas para registro da informação sobre a clientela atendida apresentam diferenças em relação à qualidade no registro das informações e semelhanças quanto ao cliente atendido. O NIS Alvorada I apresentou percentuais maiores em relação a ausência de registros que os outros dois núcleos, bem como uma maior ilegibilidade das HD registradas.

A ausência nos prontuários do registro das $\mathrm{HD}$, associada ao percentual elevado de diagnósticos ilegíveis, mesmo após uma intervenção educativa específica para melhorar a qualidade dos registros, demonstra um entrave importante para análise de qualidade a partir dos mesmos e o impacto limitado da intervenção realizada. Em decorrência disto, a possibilidade da identi- 
ficação do perfil nosológico da clientela atendida e, também, a avaliação da assistência para patologias específicas foram prejudicadas. Cabe destacar que no caso presente os percentuais encontrados sobre legibilidade e falta de informação foram superiores aos encontrados por Modesto et al. (1992), Lotufo \& Duarte (1987) e Radaelli et al. (1990). A qualidade do registro continua constituindo-se em um obstáculo sério à avaliação.

Vários autores apontam a inadequação da CID, originalmente desenvolvida para mortalidade, para registro de morbidade ambulatorial como possível fator limitante no monitoramento dessa. Mendonça et al. (1990: 304), aponta uma inadequação entre a CID e processo de diagnóstico ambulatorial, afirmando que essa decorre dos próprios limites da classificação. "São exemplos significativos desta afirmação: a insuficiente caracterização do estado normal (Pediatria); a dificuldade de incorporação de conclusões nosológicas analíticas (Psiquiatria) e a não captação da dimensão social da doença".

O ideal para a codificação seria que o médico anotasse o diagnóstico e este posteriormente seria codificado por um profissional treinado. A inexistência de profissionais treinados, e também a ilegibilidade dos diagnósticos nãocodificados pelos médicos impossibilitou o uso desse procedimento.

A procedência da clientela foi relativamente fácil de monitorar, no entanto, considera-se que eventuais falsificações no endereço residencial podem diminuir a validade das conclusões referentes a esta questão.

A análise de "resolutividade", mensurada pelo percentual de registros nos prontuários de usuários encaminhados externamente, também pode ser de baixa confiabilidade, se houve sub-registro de encaminhamentos, o que não foi possível verificar nesta pesquisa. Este indicador também não reflete plenamente se a unidade foi capaz de resolver o problema do usuário.

A tentativa de elaboração de indicadores de cobertura a partir dos prontuários, ou de dados de produção exige a existência de uma fonte fidedigna sobre a composição demográfica da população adscrita às unidades. As duas alternativas são o uso de dados censitários ou a reali- zação de censos domiciliares das áreas de abrangência dos núcleos.

No entanto, os serviços tradicionalmente operam, utilizando uma razão entre a produção da intervenção (consultas a menores de um ano ou gestantes, ou no máximo, distinguindo entre as primeiras e subseqüentes) e uma população alvo estimada. Este método pode levar a super ou subestimativas de dados de cobertura devido a erros no denominador. O uso de dados de produção total sem especificar, por exemplo, sexta consulta de puericultura, ou oitava de pré-natal conduzem a médias de coberturas que podem esconder a existência de usuários freqüentes, bem como, de grupos descobertos, ou de apresentar "coberturas" acima de 100\%. Neste trabalho dispunha-se apenas de informações de tipo de consulta e populações estimadas. Ainda assim, entende-se que é factível para as unidades ambulatoriais, que se propuserem, organizar sua coleta de informação de forma a permitir um cálculo mais acurado de cobertura. Ficou evidente, neste estudo, que os melhores níveis de cobertura estavam sendo atingidos no setor de puericultura enquanto a assistência geral e do pré-natal atigiram níveis menores.

A única referência para os serviços sobre concentração de consultas médicas é o repasse de recursos do Sistema Único de Saúde (SUS) relativo a duas consultas habitante/ano, e sabendo que esse parâmetro já foi suplantado pela Secretaria de Saúde de São Paulo, que utiliza 2,8 consultas habitante/ano, e pela própria medicina de grupo, cujo parâmetro utilizado encontra-se hoje na faixa de quatro consultas/ habitante/ano (Médici, 1991). Se os três NIS atingissem uma concentração de quatro consultas/habitante/ano teria se esperado um número de consultas mensais da ordem de 13 mil, o observado foi de 6.488. O encontrado no presente levantamento, apesar de relativo a cliente atendido no mês de outubro e não a habitante, aponta a necessidade de se realizarem discussões junto às equipes com a finalidade de construir parâmetros próprios de acordo com a capacidade dos serviços e necessidades da população, o que possibilitaria um planejamento mais adequado da oferta de recursos.

Sabe-se que numa rede básica assistencial a 
equipe de enfermagem desempenha papel relevante, pois é a que responde pelo maior volume dos atendimentos. No presente levantamento observou-se um número reduzido de registros por parte das enfermeiras, o que pode estar relacionado com a tendência de o enfermeiro estar cada vez menos vinculado às atividades assistenciais e mais vinculado às atividades administrativas (Alves, 1989). De outro modo, isto pode representar sub-registro das consultas de enfermagem.

Os dados encontrados sobre o registro de atendimentos de enfermagem também foram inferiores ao esperado. No caso do presente estudo esperava-se algo em torno de 12.976 atendimentos, levando-se em consideração apenas o número de consultas médicas registradas, sugerindo que os atendimentos estão desvinculados das demais atividades, ou então que a equipe de enfermagem não registra habitualmente o que realiza.

Em relação aos atendimentos odontológicos e psicológicos observou-se que os mesmos são registrados em prontuários diferentes e que esses não são integrados aos sistemas dos outros serviços, funcionando com normas e registros próprios, muitas vezes desconhecidas pelo restante dos profissionais.

Outros aspectos de qualidade, não previstos na elaboração original desta pesquisa, tais como, qualidade do processo de atendimento, produtividade e eqüidade, eventualmente seriam passíveis de estudo a partir de prontuários. Por exemplo, em relação a eqüidade, seria possível formular indicadores específicos, desde que exista condições de identificar subgrupos de população a serem estudados e realizar comparações da atenção recebida. Hoje nos prontuários existe espaço para o preenchimento de endereço residencial, situação de ocupação (empregado/desempregado), idade e sexo como variáveis potenciais de estudo. Já indicadores de impacto, como satisfação do usuário e trabalhador, ou modificações no quadro de saúde, demandariam desenhos e metodologias específicas para sua apuração.

Enfim, esta análise demonstra que os prontuários nos serviços de saúde estudados funcionam precariamente como fonte de informação, tanto para uso interno como para avaliação.
Servem, sim, como indicativo da qualidade precária da atenção, se entende-se que a qualidade da informação é relacionada com a qualidade do cuidado. Apesar destas insuficiências, foi possível extrair indicadores de qualidade dos prontuários, sendo necessário para garantir confiabilidade destes indicadores melhoras significativas na qualidade do preenchimento. Um registro melhor teria permitido o desenvolvimento de indicadores de avaliação de processo e de resultados mais aprofundados.

Informação isolada não é a solução dos problemas de qualidade, mas busca-se através dela iniciar uma discussão junto às equipes, sobre o que se está produzindo na rede. A informação é, geralmente, produto de uma atividade que possui significado e sentido especial dentro do processo de trabalho, sua coleta é vista como tarefa secundária imposta pelos planejadores, elaborada centralizadamente, mantendo alijados os trabalhadores de qualquer participação, o que a faz parecer estranha e desnecessária.

Profissionais da rede freqüentemente rejeitam a discussão de seu trabalho sem uma vinculação direta e imediata a sua prática. Nesse sentido, reforça-se a idéia de que as informações levantadas diretamente a partir de suas práticas possam facilitar a reflexão proposta. Havendo por parte dos envolvidos desconfiança acerca da confiabilidade de muitos dados, a essa poderão contrapor-se as informações que foram por eles mesmos produzidas, facilitando o início do diálogo.

$\mathrm{O}$ uso de informações provenientes dos serviços pode contribuir para a avaliação na perspectiva de promover a garantia de qualidade. Tais esforços não se encerram quando se define a sua abordagem teórica ou se estabelece os critérios técnicos da medida de qualidade, pois esses demandarão intervenções no campo da prática e, a partir dessas, espera-se que ocorram modificações, na qualidade dos serviços ofertados à população.

\section{AGRADECIMENTOS}

À professora Lynn Silver pela orientação e leitura crítica. À Secretaria Municipal de Saúde de Maringá, em especial à Esmavete Rocha pela colaboração. 


\section{RESUMO}

\section{SCOCHI, M. J. Indicadores da Qualidade dos Registros e da Assistência Ambulatorial em Maringá, (Estado do Paraná, Brasil), 1991: Um Exercício de Avaliação. Cad. Saúde Públ., Rio de Janeiro, 10 (3): 356-367, jul/set, 1994.}

Buscou-se indicadores de qualidade da assistência passíveis de análise a partir de registros rotineiros na rede ambulatorial de serviços de saúde da Secretaria Municipal de Saúde de Maringá, Paraná (Brasil). Foram avaliados os atendimentos realizados em três Núcleos Integrados de Saúde (NIS) com o objetivo de verificar se o que foi registrado permite a formulação de indicadores de qualidade. Pesquisou-se todos os prontuários dos 7.813 pacientes atendidos durante o mês de outubro de 1991. Foram formulados indicadores de qualidade de registro, procedência da clientela, demanda, cobertura e resolutividade por unidade de saúde. Em 49,7\% dos prontuários, as hipóteses diagnósticas (HD) estavam ausentes e em $14,8 \%$ haviam HD ilegíveis; $78 \%$ dos atendimentos foram destinados a moradores que residiam na área de abrangência das unidades; a cobertura de gestantes foi baixa $37 \% ; 70 \%$ das crianças menores de um ano da comunidade foram atendidas no mês, e as pessoas atendidas tiveram em média três consultas médicas no ano precedente, $4,3 \%$ dos prontuários dos usuários atendidos apresentava registro de encaminhamentos para outros serviços. Os prontuários nestes três núcleos funcionam precariamente como fonte de informação, tanto para uso interno como para avaliação. Apesar destas insuficiências foi possível extrair alguns indicadores de qualidade, sendo necessário, melhoras significativas na qualidade do preenchimento, para sustentar avaliações mais aprofundadas.

Palavras-Chave: Planejamento em Saúde; Avaliação de Serviços; Qualidade; Informação em Saúde; Assistência Ambulatorial

\section{REFERÊNCIAS BIBLIOGRÁFICAS}

ALVES, M., 1989. O Enfermeiro Enquanto Gerente. Anais $5^{\circ}$ Ciclo Nacional de Administração em Enfermagem. Maringá: Universidade Estadual de Maringá. (Mimeo.)

CORDONI Jr., L. \& BERTONE, A. A., 1992. Melhoria do desempenho dos serviços públicos de saúde: pontos de estrangulamento e caminhos para a superação. Cadernos $d a$ Nona, 2: 175-179.

DONABEDIAN, A., 1984. La Calidad de la Atención Médica: Definición y Método de Evaluación. México: La Prensa Mexicana.

IBGE (Instituto Brasileiro de Geografia e Estatística), 1991. Censo Demográfico de 1991 - Paraná. Sinopse Preliminar. Brasília: IBGE. (Mimeo.)

IPARDES (Instituto Paranaense de Desenvolvimento Social), 1990. População Censitária de Maringá em 1990, por Zona, Sexo e Faixa Etária. Base de Dados do Estado do Paraná. Maringá: Ipardes. (Mimeo.)

LOTUFO, M. \& DUARTE, E. C., 1987. Avaliação dos serviços de saúde do Município de Cácere, MT (Brasil): contribuições à programação local. Revista de Saúde Pública, 21: 427-438.

MÉDICI, A. C., 1991. Descentralização e informação em saúde. Revista Planejamento e Políticas Públicas, 5: 05-31.

MENDONÇA, M. C. L. G.; SOUZA, M. S. L.; NEHMY, R. M. Q.; CUNHA, E. G. A.; BICHUETTI, J. A. N. \& SANTOS, A. F. 1990. Avaliação de dados nosológicos em prontuários ambulatoriais. Cadernos de Saúde Pública, 6: 293-305.

MS (Ministério da Saúde); SNPES (Secretaria Nacional de Programas Especiais de Saúde); DINSANI (Divisão Nacional de Saúde Materno Infantil); INAMPS (Instituto Nacional de Assistência Médica da Previdência Social), 1984. Programa de Assistência Integral à Saúde da Criança. Brasília: MS. (Mimeo.)

MODESTO, M. G.; MOREIRA, E. C. \& ALMEIDA-FILHO, N., 1992. Reforma sanitária e informação em saúde: avaliação dos registros médicos em um distrito sanitário de Salvador, Bahia. Cadernos de Saúde Pública, 8: 62-68. 
RADAELLI, S.; TAKEDA, S. M.P.; CIMENO, L. I. D.; WAGNER, M. B.; KANTER, F. J.; MELLO, V.M.; BORGES, J.C. \& DUCAN, B. B., 1990. Demanda de serviço de saúde comunitária na periferia de área metropolitana. Revista de Saúde Pública, 24: 232-240.

ROCHA, J. S. Y.; SIMÕES, B. J. G. \& FOSTER, A. C., 1991. Informações em saúde para o SUS. Um marco de referência para sua democratização. Revista de Medicina de Ribeirão Preto, 24: 186-191.

SILVER, L., 1992a. Aspectos metodológicos em avaliação dos serviços de saúde. In: Planejamento Criativo: Novos Desafios em Políticas de Saúde (E. Gallo, F. J. U. Rivera \& M. H. Machado, orgs.), pp. 195-210, Rio de Janeiro: Relume-Dumará.
, 1992b. Quality Assurance in Health Care Issues in Health Care Delivery and Finance in Brasil. Rio de Janeiro: Escola Nacional de Saúde Pública. (Mimeo.)

VUORI, V. H., 1982. Quality assurance of health services: concepts and methodology. Public Health in Europe, 16: 05-28. (WHO Regional Office for Europe Copenhagen) 